\title{
Fully automatic CNC machining production system
}

\author{
Jeng-Dao Lee , Cheng-Wei Tsai-Lin, Yi-Cheng Lee, Min-Che Liu, and Li-Yin Chen \\ National Formosa University, Automation Engineering Department, 632 Yunlin County, Taiwan
}

\begin{abstract}
Customized manufacturing is increasing years by years. The consumption habits change has been cause the shorter of product life cycle. Therefore, many countries view industry 4.0 as a target to achieve more efficient and more flexible automated production. To develop an automatic loading and unloading $\mathrm{CNC}$ machining system via vision inspection is the first step in industrial upgrading. CNC controller is adopted as the main controller to command to the robot, conveyor, and other equipment in this study. Moreover, machine vision systems are used to detect position of material on the conveyor and the edge of the machining material. In addition, Open CNC and SCADA software will be utilized to make real-time monitor, remote system of control, alarm email notification, and parameters collection. Furthermore, RFID has been added to employee classification and management. The machine handshaking has been successfully proposed to achieve automatic vision detect, edge tracing measurement, machining and system parameters collection for data analysis to accomplish industrial automation system integration with real-time monitor.
\end{abstract}

\section{Introduction}

The profit of automated production comes from a large machining of single species. By considerable production can be brought to the benefit of high effect, high yield, and low cost. The production pattern of low-volume customized is conferenced to uneconomic, slow payback period and difficult of production scheduling. The change of consumer's habit results in the product life cycle become faster. The importance of CTO is displayed at this time [1]. However, the pattern of the low-volume customized can't avoid complex pre-operation. Besides, the sudden situations are like the customer temporary increasing quantities and the customer return the unqualified product, etc. From the above, the product output the low yield if the pre-operation is more and more frequently. The problem deeply troubles the manufactures with the necessary waste. Therefore, Germany proposes the plan of industry 4.0.

The purpose of Industry 4.0 was built a highly flexible production and service model of customization and quantization, with real-time interactions between people, products, and devices during the production process. For example, a factory that accepts consumer orders and directly produces, and ships the required product will dispense of separate sales and circulation channels, which will have a strong impact on the traditional e-commerce sales model. Industry 4.0 will affect not only industry of Germany, or even international industrial development but will become a driving force [2].

Take the countries of relevant policy of Industry 4.0. For example, Germany has developed a strategic plan to implement Industry 4.0. The main points of this plan can be summarized as: building a network, researching two major themes, realization of three integrations, and achievement of eight planning objectives [3]. Japan has proposed a new strategy for the robot policy. It focuses on the coexistence of man-machine for the future factory. The technical strategy predominantly is based on the systematic integration that developing sensors system, controller system, driver system, cloud computing, and artificial Intelligence of robot. The method will use in the requirement of aged society and revitalized the economy of manufacturing. China proposed the policy of made in China 2025. It based on the intelligent manufacturing developing the new generation of mobile communications, internet of things, cloud computing and other industry. The policy will achieve mechanical equipment of high level and informatization of the manufacturing [4].

In view of above, industry 4.0 demonstrates the fundamentals of future manufacturing technology. The device must have the foundation of digital information and matched the hardware and software in order to optimize the flow of production. The production line achieved the optimization of the product By instantly transmit the communication between production and facility.The method will help manufacturers to product life management if industry 4.0 be achieved it. The manufacturer can advance planning of product design, production, and maintenance routine. Firms shortened the time to market of the product by the planning of intelligent manufacturing. Manufacturers can be a more accurately grasp the marketing of the product [5]. 
In conclusion, Industry 4.0 must rely on smart manufacturing, smart production, and smart product in series when the production is achieved the performance. In the industrial value chain, the $\mathrm{CNC}$ controller will undoubtedly be the hub because it can control machines and communicate between factory and the internet. The method not only thinks outside the box of CNC industry, also it brings the chance to upgrade for the machine-tool industry in Taiwan.

The research is to explore actual demand based on the machine-tool industry. The approach uses the $\mathrm{CNC}$ controller, servo motor, variable-frequency Drive, intelligent sensors, PLC, and manipulators. CNC controller accomplished the connection between devices through the serial transmission (RS485). At the same time, the overall system of the important data instantly transmits to the computer by Ethernet. Using the SCADA software from DELTA ELECTRONICS, INC. developed the man-machine interface to monitoring. It will alert and inform the relevant departments when the machinespecific parameter is abnormal.

\section{System of Description}

System block diagram shows as Figure 1. The CNC controller is the main controller in the system. Servo motors in the moving machining platform are connected the CNC controller via DMCNET. The parameters of other equipment are collected to SCADA software by RS485 and RS232 protocol. User can monitor machine on the computer by Ethernet. The PLC (programmable logic controller) sends command to the servo motor which is on the conveyor via CANopen protocol, and sends ASCII string to the manipulator (Six-axis articulated robot arm) using RS232 protocol. User can immediately get machining data via the computer. The real-time monitor was accomplished the integration of the multi- automated system by SCADA software.

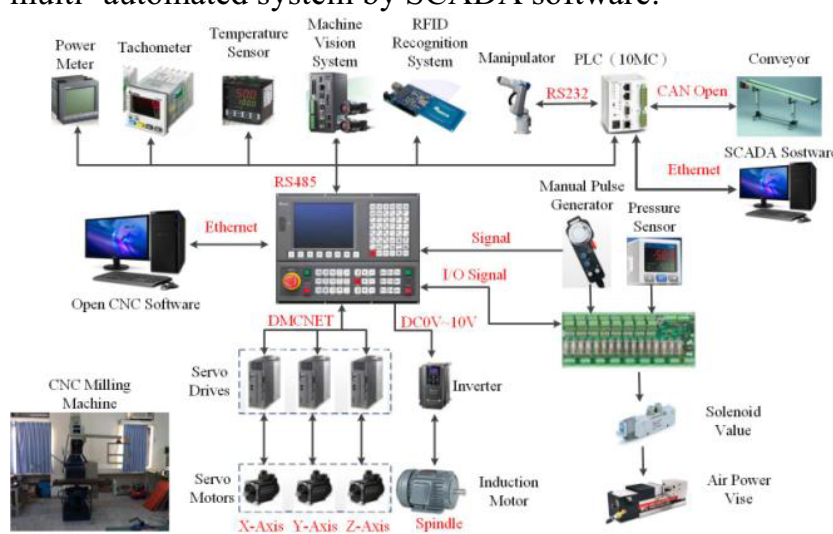

Figure 1.System structure

The manipulator is used to loading and unloading the material to accomplish automatic machining. The operational flow chart can be separated into three parts: vision inspect, manipulator and CNC machining.

The vision inspection conveyor operational flow chart is shown in Figure 2. The vision inspects the material whether on the conveyor or not when the conveyor is moving.

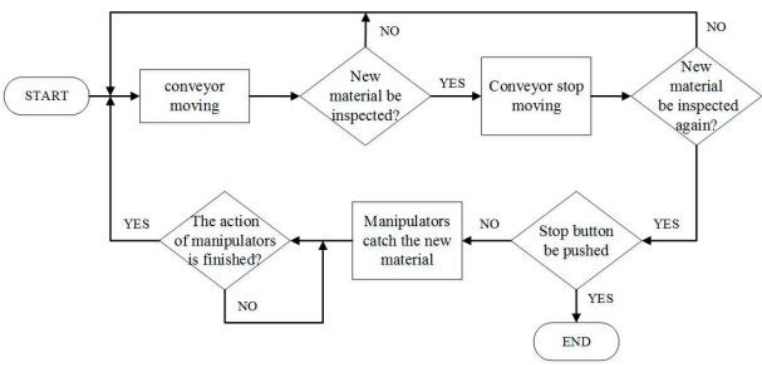

Figure 2.Vision inspection conveyor operational flow chart

The manipulator operational flow chart as Figure 3, the manipulator will catch the new material when the new material vision has been detected.

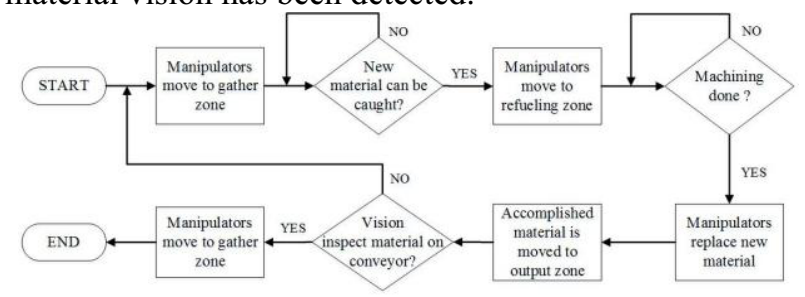

Figure 3.Manipulator operational flow chart

CNC miller is adopted in the fully automatic CNC machining production system and the operational flow chart is shown in Figure 4. CNC miller will move to the zone of edge finder and finding the edge of $\mathrm{X}$ coordinate. After done the inspection of edge, CNC miller began to the machining the material when it back to the origin. After machining is finished, the $\mathrm{CNC}$ will be waiting for manipulator to change the material.

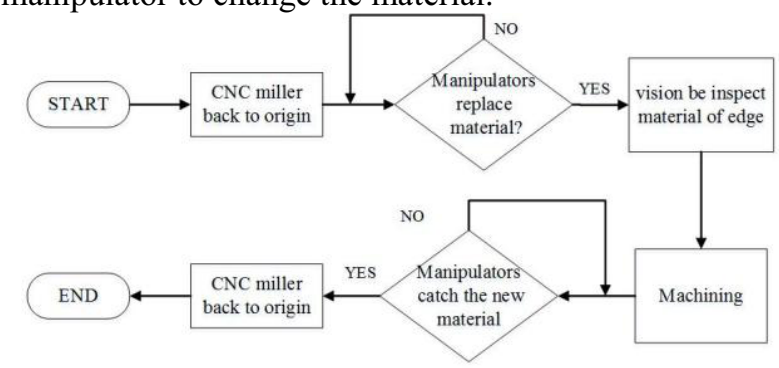

Figure 4.CNC miller operational flow chart

\section{Vision Inspection}

In the current industry of the automatic factory, the method is often replaced man-resource what using the manipulators to loading and unloading. In order to achieve the effect of automatic loading and unloading go well with the manipulators and vision inspection.

The following sections will point out the introduction for respective to edge finder and object finder.

\subsection{Material Finder System}

The research will go with the manipulator to loading and unloading so as to complete the automated production line. The position of the object is different when the conveyor was delivered the material every time. In order 
to avoid this problem, the vision inspection will go to determine the current position of materials.

Figure 5 is shown in the interface of the material finder. The analytical data of $\mathrm{X}$-axis position, $\mathrm{Y}$-axis position, and angle. The relevant parameters will be transferred to CNC controller when new material was been inspected via the material finder. In view of above, the transmission is important among the devices for the automatic production line. Next, following will discuss the delivery method with regard to the vision inspection system and the manipulator.

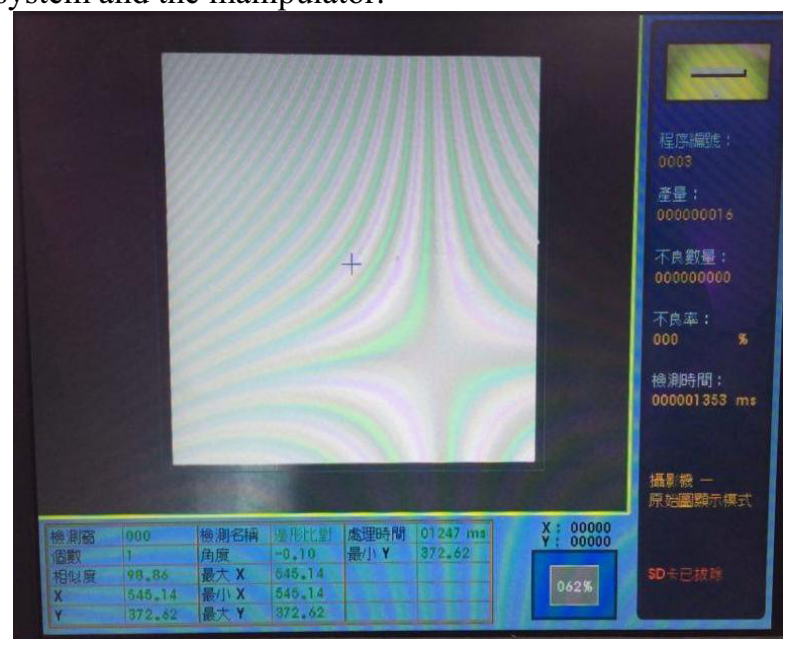

Figure 5.Interface of detection position

As Figure 6 shown, the slaves of devices delivered command by $\mathrm{CNC}$ controller, and slaves will act according to the special command by the $\mathrm{CNC}$ controller.

The vision inspection system will store numerical value to the internal memory when shooting was completed. The CNC controller will catch the numerical value from the internal memory of vision inspection system. At the same time, CNC controller sends the relevant parameter to the multi-axis controller of PLC. The numerical value changes to the ASCII of character string when the multi-axis controller received it. Finally, the changed character string will command manipulator to act. With the method, it achieves to the communication between the devices.

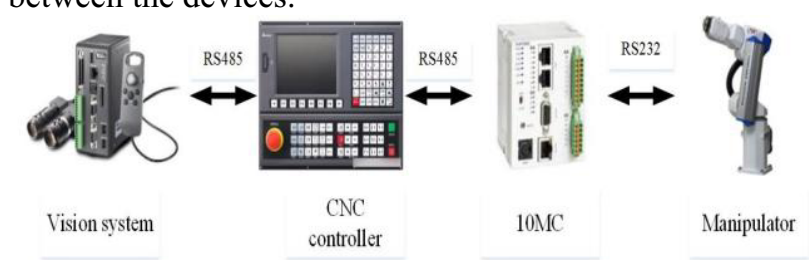

Figure 6.The communication architecture of vision system

\subsection{Edge Finder System}

The act will lead material to deviation when the pressure vise has gone on the work of clamping. So the machining has the different origin every time, and then makes the precision to reduce.

Therefore, the research was look for the edge of material by vision inspection in order to improve the precision of machining. As Figure 7 shown, the parameter from zone of edge finder has transmitted to $\mathrm{CNC}$ controller for compensating. The vision inspection system will compensate the error coordinate origin when automatic loading results in the different position.
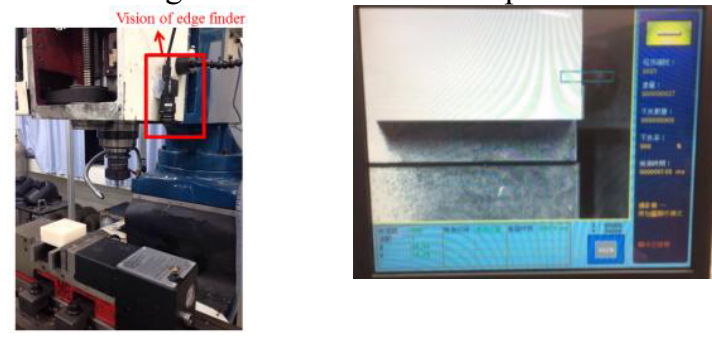

Figure 7(a).position of camera

Figure7(b). Picture of edge finder

\section{Monitor and Control}

In order to achieving the function of the system to supervise, the following will explore the man-machine interface of Open CNC and SCADA.

In this part of Open $\mathrm{CNC}$, it added to the function of the send email of alarm and real-time surveillance system. In the function of the send email, the Open $\mathrm{CNC}$ will notify the relevant department by the email when the machine have happened alarm. In the function of realtime surveillance system, it monitored the current image via webcam. The foregoing method will increase work efficiency.

In this part of SCADA software, the method of transmission connects the programmable logic controller (PLC) by the Ethernet. PLC will deliver CNC parameters like temperatures, currents, voltages...etc. to SCADA software. By the above method, achieving the purpose of prevented to failure in advance and improved efficiency. The users can beforehand prevent abnormal happened such as the tool wear and the machine is out of order...etc. by analysis parametric.

Figure 8 shown the status of the production process, the picture of SCADA will display where the current position of the mechanical production line.

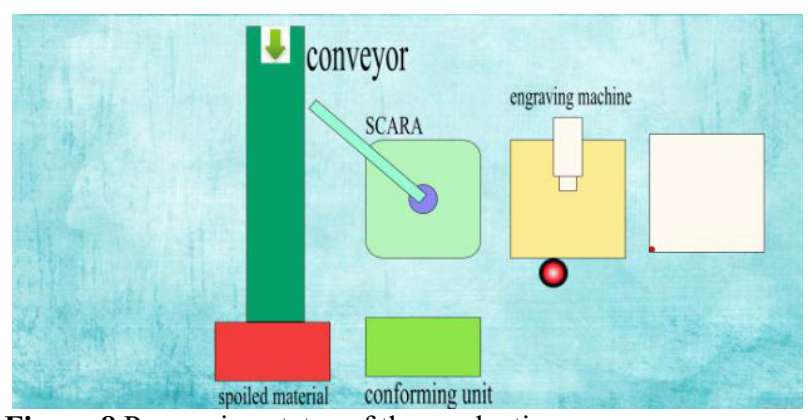

Figure 8.Processing status of the production process

Figure 9 shows the supervisory system and displayed the most important parameter on the human-machine interaction from PLC (10MC) deliver to CNC controller. At the same time, the system also planned the real-time display for the material detection region. The Figure 9 demonstrated the current position of material in the range of the camera. The parameter delivered to the real-time 
graph from PLC (10MC), and it shows the current position on the conveyor.

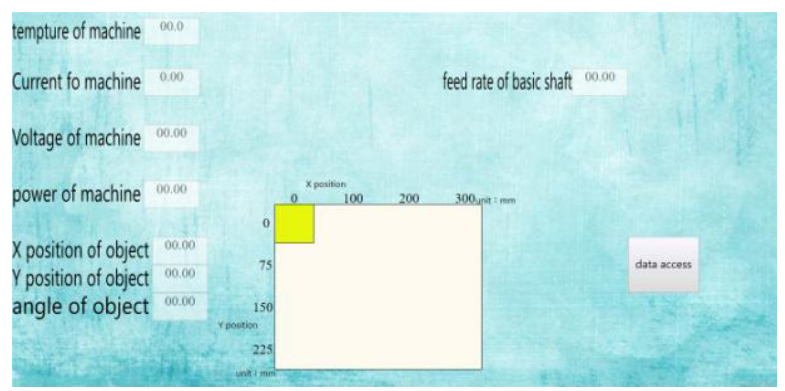

Figure 9.The supervisory system

As Figure 10 shown the interface of alarm records, the function will record the error and the form can export to excel. Users can focus on the detection into the machine according to the special alarm of history record. The method will promote the effect of work.

\begin{tabular}{|c|c|c|c|c|c|c|c|c|}
\hline A & B & c & D & E & E & G & H & I \\
\hline Alert notification & time of happened & time of reseting & $\begin{array}{l}\text { alam } \\
\& \\
\text { resulting }\end{array}$ & alent level & alert type & $\begin{array}{c}\text { currert } \\
\text { temperature }\end{array}$ & $\begin{array}{l}\text { nommal } \\
\text { range }\end{array}$ & source \\
\hline 2 temperature is abnormal & $2016 / 11 / 3013: 17: 04$ & & alarm & high & general & 37 & 30 & JDLab_kachu_PC \\
\hline 3 temperature is abnormal & $2016 / 11 / 3013: 18: 36$ & $2016 / 113013018-59$ & resulting & high & general & 27 & 30 & JDLab_kachu_PC \\
\hline 4 temperature is abnormal & $2016 / 11 / 3013: 19: 03$ & $2016 / 11 / 30$ 13:19:11 & resulting & high & general & 29 & 30 & JDLab_kachu_PC \\
\hline 5 temperature is abnormal & $2016 / 11 / 3013: 19: 15$ & $2016 / 11 / 30$ 13:19:22 & resulting & high & general & 26 & 30 & JDLab_kachu_PC \\
\hline 6 temperature is abnormal & $2016 / 111 / 30$ 13:37:40 & & alamm & high & general & 43 & 30 & JDLab_kachu_PC \\
\hline
\end{tabular}

Figure 10.Function of alarm records

Figure 11 shows the control software interface (Open $\mathrm{CNC}$ ). The software is specially developed for $\mathrm{CNC}$ controller for Delta Electronics, Inc. The method provided the more choice for operation.

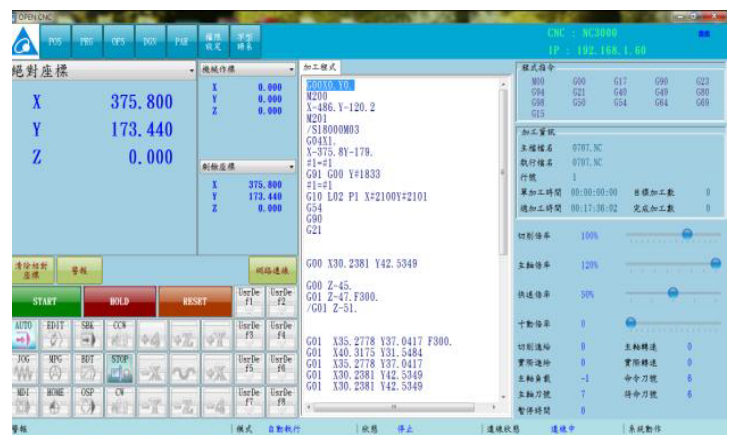

Figure 11.Open $\mathrm{CNC}$ software

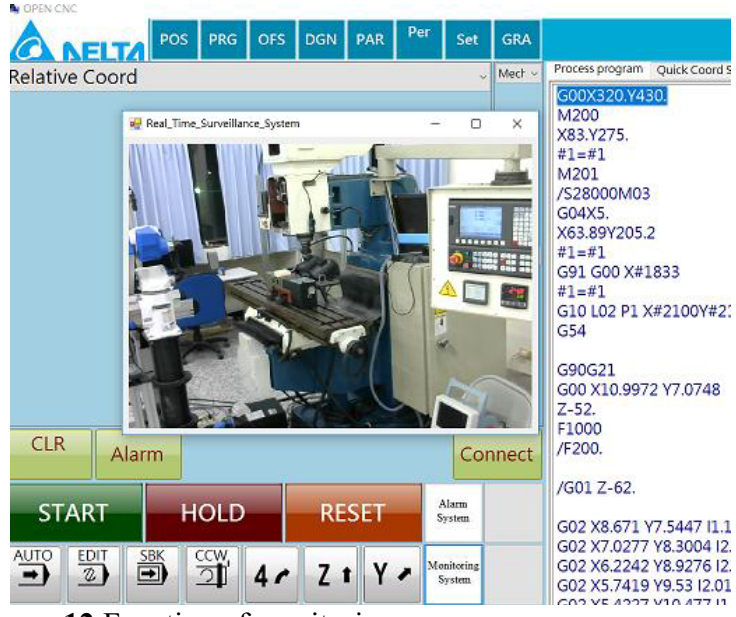

Figure 12.Function of monitoring
Figure 12 shows the real-time surveillance system. The function surveyed the situation of the current scene. Users can know whether have happened the situation of error, and they can immediately aid the field employees.

Figure 13 shows the function of sending a mail. The mail will be sent to the designated department when there is an error happened on the machine. The designated department resolve the error of machine in best time. The function can reduce the time of resolve the alarm and increase the work efficiency.

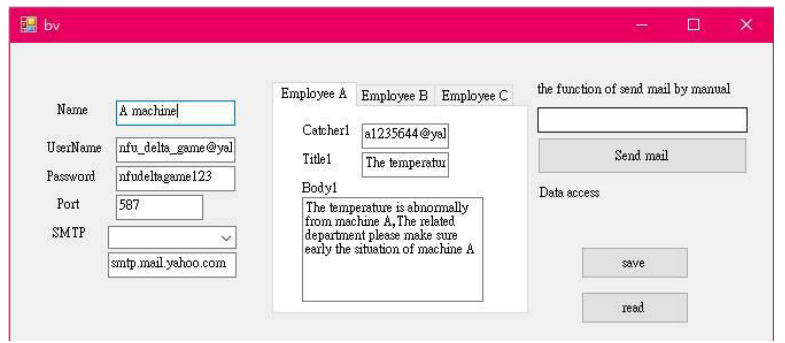

Figure 13.Function of send mail

The mail also set up the three patterns of Yahoo, Hotmail, and Gmail. Users can set up the pattern of transmission according to the habit of the company. The method achieves to the transmission of Extranet. It can deliver the message to the designated company when the machine happened the particular error.

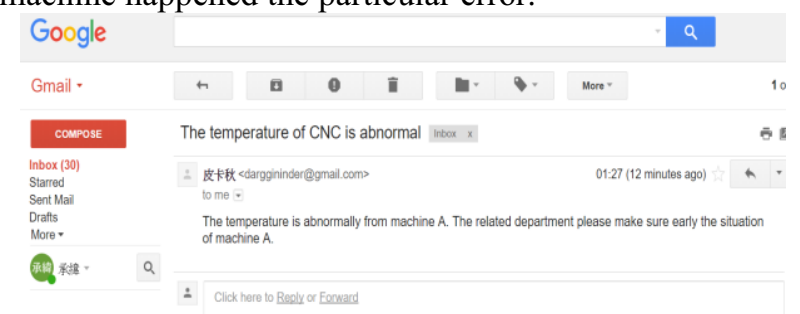

Figure 14.Warning email Gmail

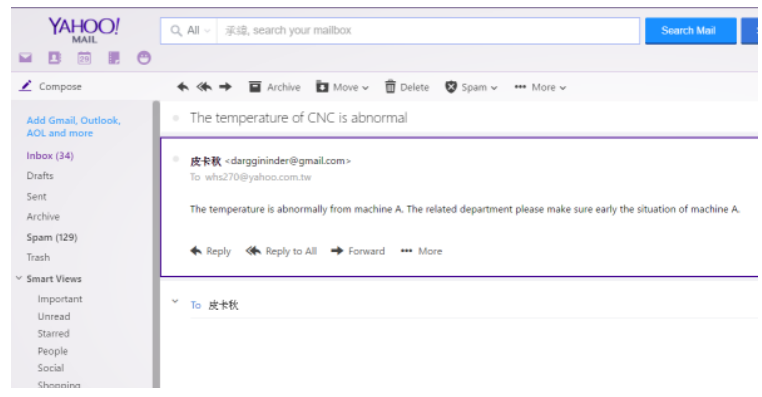

Figure 15.Warning email Yahoo

\section{Experiment Result}

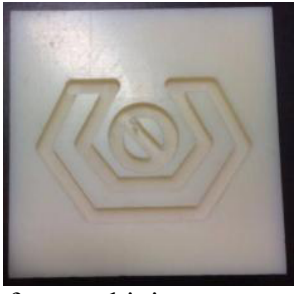

Figure 16. Material after machining

The using material is $70 \mathrm{~mm} * 70 \mathrm{~mm} * 30 \mathrm{~mm}$ of ABS. With developed the automatic loading and $\mathrm{CNC}$ machining 
system via vision inspection. Users can process lots of same products, and they can alter the project when the requirement is different. As Figure 16, the material is completed machining by the automatic production line.

As Figure 17, the air pressure-clamp on the manipulator to loading and unloading automatically when the vision inspection system inspects material position and angle from the machining platform and the conveyor. CNC miller can machine the graph accurate by the edge find from vision inspection system.

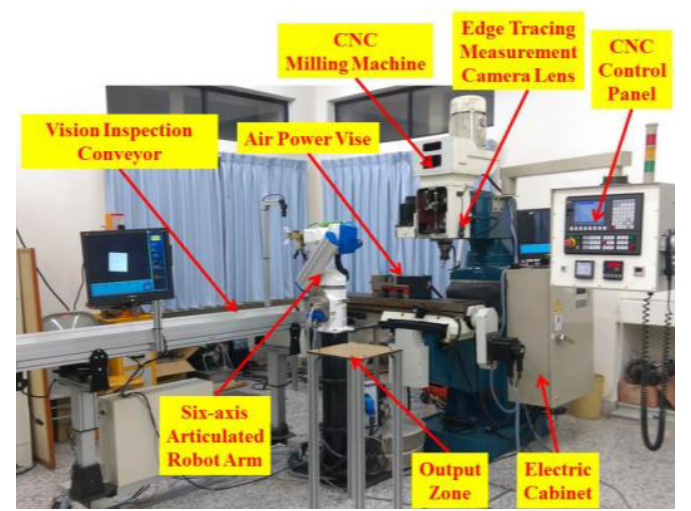

Figure 17.Equipment Instruction

As Figure 18, compare the costing time between human and vision system in edge tracing. It displayed the edge tracing by human cost around 60 to 100 seconds. If the production line use edge tracing by vision, after filming, coordinate calculate and $\mathrm{CNC}$ bed moving just only cost 10 seconds to finish, and the time of machining is standardization. According to above of result, the project chosen approach of the vision inspection is more effective.

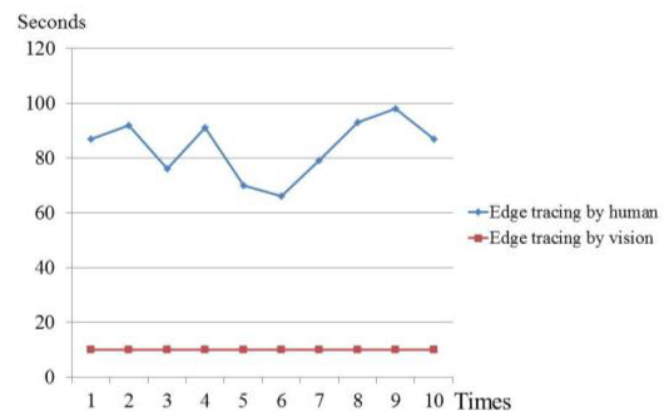

Figure 18.Edge tracing cost time between human and vision comparison chart

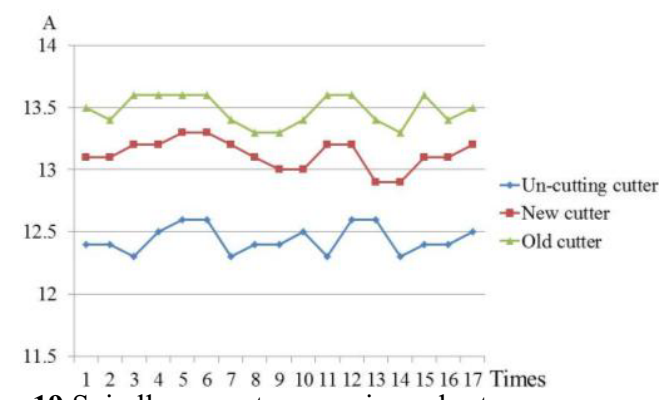

Figure 19.Spindle current comparison chart

In this research, SCADA software is used to get spindle current value to analysis - un-cutting, new cutter and old cutter in the same machining situation. As Figure 19, we can know current value difference among new cutter, old cutter and un-cutting cutter. If the cutter was worn, the current value is bigger than a new cutter. So users can know the cutter status and change cutter at the suitable timing by the parameter of current.

\section{Conclusions}

In this research, an automatic loading and unloading $\mathrm{CNC}$ machining system via vision inspection had been developed. CNC controller is the core of automatic production line, and it completed the communication among all devices. In the system of the object finder, the position and angle of incoming materials will be inspected and saved in the registers when the material photograph is passing vision inspection on the conveyor. The CNC controller sends those data to the programmable logic controller (10MC) by RS485 to send the command to control the manipulator, conveyor. The project completed an automatic production line with high-speed edge finding, the material which is on CNC miller moved to edge finding zone and it will be inspect after manipulators placing materials.

At the same time, the SCADA software collected the real-time data from the related sensors when the automatic production line is processing. The function of real-time monitor and alert email notification were built by Open CNC. The related worker will receive alert email or notification when alarm happened so that they can troubleshoot immediately. With SCADA and Open $\mathrm{CNC}$, the alarms are prevented and reduce the repair times so that the production efficiency will be increased.

\section{Acknowledgment}

The authors would like to acknowledge the financial support of the Ministry of Science and Technology of Taiwan, R.O.C. through grant number MOST 105-2221E-150-038.

\section{References}

1. "The synopsis of FMS be proposed it, "2011(in Taiwan)

2. J. Wan, H. Cai, and K. Zhou, "Industrie 4.0: enabling technologies." In Intelligent Computing and Internet of Things (ICIT), 2014 International Conference on. IEEE, 2015, pp. 135-140.

3. W. Luo, "Germany industrial 4.0 strategic enlightenment to China's industrial transformation," Fiber Reinforced Plastics/Composites, pp. 125-128, 2014 (in Chinese).

4. "Executive Yuan provide the developing scheme of industry 4.0, "2015(in Taiwan)

5. Y. Wang, "It from industry 4.0 is observed the challenge of productivity 4.0", 2015 (in Taiwan).

6. LI-JING industrial Co., Ltd-conveyor . 2016(in Taiwan).

7. DELTA ELECTRONICS, INC. 2016 (in Taiwan).

8. J. Chen, "the sever motor adjusts it from $\mathrm{CNC}$, "May. 2015 (in Taiwan). 